\title{
Desempenho neuromotor, prematuridade e baixo peso à nascença
}

Neuromotor performance, prematurity and low birth weight

\author{
Alfredo Alexandre Tomé Lopes \\ Go Tani ${ }^{2}$ \\ José António Ribeiro Maia
}

1. Universidade do Porto. Faculdade de Desporto. Laboratório de Cineantropometria e Estatística Aplicada. Porto. Portugal.

2. Universidade de São Paulo. Escola de EducaÇão Física e Esporte. Programa de Pós-Graduação em Educação Física. São Paulo, SP. Brasil.1

Recebido em 23/04/10 Revisado em 10/08/10 Aprovado em 15/09/10
Resumo - Parece ser consensual que as influências intrauterinas durante o desenvolvimento humano podem ter implicações a longo prazo. Há quem avance a hipótese da indução de alterações no ciclo de vida de um indivíduo provocando lesões, algumas irreversíveis. Os objectivos deste trabalho foram: (1) chamar a atenção para a problemática dos efeitos à distância do desempenho neuromotor, prematuridade e baixo peso à nascença em crianças e jovens; (2) apresentar aspectos relevantes do quadro conceptual da programação fetal e da sua relevância interpretativa na insuficiência de desempenho motor e coordenativo. Realizou-se uma pesquisa na base de dados Pubmed por meio de uma estratégia de busca com palavras-chave e suas possíveis combinações e foram seleccionados os estudos (transversais, longitudinais e caso-controlo) sobre avaliação da performance motora e da programação fetal. Na população europeia, os valores dos défices motores variaram entre $4 \%$ e $51 \%$. A população norte americana apresentou valores que se situam entre 4\% e 56\%. Uma pesquisa realizada com a população da Austrália encontrou um défice de coordenação motora de 9,5\%. Não existe concordância absoluta acerca da sua extensão, reversibilidade e significado face à enorme variabilidade dos valores dos défices. Não são conhecidos aspectos dos eventuais mecanismos que, em condições adversas no desenvolvimento fetal, induzirão problemas no desenvolvimento neuromotor de crianças, jovens e adultos, e que não possam ser "compensados" com estimulação sensório-motora.

Palavras-chave: Baixo peso à nascença; Performance neuromotora; Programação fetal.

Abstract - There seems to be consensus that intrauterine influences during human development have long-term implications. The hypothesis has been raised that alterations in an individual's life cycle provoke lesions, some of them irreversible. The objectives of this study were: (1) to call attention to the problem of distance effects of neuromotor performance, prematurity and low birth weight in children and youngsters; (2) to present relevant aspects of the conceptual framework of fetal programming and its interpretive relevance for deficits in motor performance and coordination. A Pubmed database search was performed using different key words and their possible combinations. Cross-sectional, longitudinal and case-control studies evaluating motor performance and fetal programming were selected. Motor deficit levels ranged from $4 \%$ to $51 \%$ in the European population and from $4 \%$ to $56 \%$ in the North American population. A study involving the Australian population reported a motor coordination deficit of $9.5 \%$. There is no absolute agreement regarding the extent, reversibility and significance of motor deficits in view of the wide variability in deficit levels. The mechanisms that will induce problems in the neuromotor development of children, youngsters and adults under adverse conditions of fetal development are unknown and cannot be compensated for by sensorimotor stimulation.

Key words: Low birth weight; Neuromotor performance; Fetal programming. 


\section{INTRODUÇÃO}

É um facto inequívoco que de todas as espécies que habitam a terra, o homo sapiens sapiens é a que tem um crescimento e desenvolvimento mais prolongados, apresentando, durante vários anos, um estado de imaturidade biológica que a obriga a uma dependência de cuidados e protecção para sobreviver.

Segundo Malina et al. ${ }^{1}$, em crianças de Baixo Peso à Nascença (BPN), a prematuridade implica alterações em diversos aspectos do seu desenvolvimento motor (DM) que são causadas, provavelmente, por eventuais atrasos no desenvolvimento do seu corpo e tecido neural.

Nascem, actualmente, em nível mundial, cerca de 25 milhões de crianças com baixo peso ${ }^{2}$. Segundo a Comissão Nacional de Prevenção da Mortalidade Infantil Norte-Americana, o aumento da percentagem de crianças com BPN dá origem, com uma incidência de 250000 por ano, a risco de problemas crónicos de saúde 3 .

O BPN foi definido pela Organização Mundial de Saúde (OMS) como o peso ao nascer inferior a $2500 \mathrm{~g}$ e a prematuridade com uma gestação inferior a 36 semanas $^{3}$. Dados epidemiológicos apontam que crianças com BPN têm 20 vezes mais chances de morrer prematuramente. Este é, de facto, um indicador de elevada importância em termos de Saúde Pública². O BPN é um importante factor de risco que pode resultar de uma gestação curta ou de uma diminuição do crescimento intrauterino. Em termos gerais, a sua prevalência nos países desenvolvidos é de 7\%, enquanto que nos países em desenvolvimento é de $16,5 \%$, sendo que na Ásia Central correspondem a 27\%. Na África sub-sariana é de 15\%, na América Central de 10\%, o mesmo que na Austrália. Os dados relativos a Portugal (2001) e Brasil (2002), segundo a OMS, situam a prevalência em $8 \%$ e $10 \%$, respectivamente ${ }^{2}$.

Nos países desenvolvidos, os avanços nas unidades neonatais contribuíram para uma sobrevida sem precedentes ${ }^{4}$, as melhorias relativas a meios técnicos, tecnologias e cuidados médicos têm vindo a aumentar a qualidade do socorro; no entanto, também aumentam a morbilidade 5 . Por outro lado, nos países em desenvolvimento com carências severas em cuidados de saúde, higiene e nutrição, observam-se elevadas taxas de crianças de $\mathrm{BPN}^{2}$.

Em 2001, 8\% de um total de 4,6 milhões de crianças nascidas nos E.U.A. foram diagnosticadas como sendo prétermo/BPN. Os custos totalizaram 5,8 biliões de dólares, representando 47\% dos cus- tos de todas as crianças hospitalizadas, com uma média de 15100 dólares e uma média da estadia de 12,9 dias. Os custos aumentam para uma média de 65600 dólares para extremo BPN (EBPN) ${ }^{6}$. Estes valores revelam o grande impacto financeiro das crianças com BPN, mas não incluem os custos da sua associação com consequências tardias do foro neuromotor ou outras.

O BPN está intimamente associado com a mortalidade e morbilidade fetal e neonatal e limita o crescimento físico e o desenvolvimento cognitivo, é reconhecido estar associado a diversos défices de natureza cognitiva e neuromotora, embora não exista concordância em relação a sua extensão e implicação face à enorme variabilidade dos valores desses défices ${ }^{4,7-11}$.

Rogers et al. ${ }^{12}$, ao avaliar 53 crianças com peso $<800$ g e gestação $<29$ semanas, verificaram que, aos 17 anos, elas tinham capacidade aeróbia, resistência, força, flexibilidade e coordenação mais baixas quando comparadas com crianças de grupo controlo (>2500g). Também apresentavam maiores dificuldades coordenativas, sobretudo, em tarefas que reclamam ritmo e cadência de execução. Estas crianças com BPN e Prematuras podem estar em risco de ter alterações do desenvolvimento por várias razões: (1) como resultado de dificuldades precoces e prolongadas; (2) por nascer com órgãos imaturos; (3) pela necessidade de tratamento em unidades de cuidados intensivos agressivos em todo o período neonatal, com adaptação ao stress, ambiente hospitalar altamente estimulante e, (4) pela falta de experiências sociais com a mãe. Segundo Barker ${ }^{13}$, as influências intrauterinas durante períodos críticos do desenvolvimento humano podem ter implicações a longo prazo. Tem sido fortalecida a hipótese de que as alterações na estrutura do corpo, na fisiologia e no metabolismo do feto podem induzir alterações no ciclo de vida de um indivíduo, tais como a emergência de diabetes tipo 2 , hipertensão, doenças cardiovasculares ${ }^{3}$, doença pulmonar obstrutiva crónica, falência renal, cancro do ovário e outras doenças ${ }^{2}$. Segundo $\operatorname{Loos}^{14}$, o aumento da prevalência destas doenças crónicas constitui uma das preocupações centrais de especialistas em Saúde Pública dos países ocidentais. Uma parte substancial da investigação sobre a etiologia dessas doenças está focalizada nos factores de risco resultantes de alterações comportamentais em resposta às modificações provenientes do modus vivendi do homem moderno.

As origens fetais têm sido estudadas, mais frequentemente, nas relações entre o BPN e diferentes 
aspectos da saúde no estado adulto, produzindo informação sólida que suporta a hipótese de Barker. O BPN é resultado, entre outros factores, de um ambiente pré-natal desfavorável.

Este texto pretende apresentar uma síntese relativamente alargada dos efeitos do BPN no desenvolvimento de crianças e jovens no que se refere a coordenação motora geral. Por coordenação motora geral entende-se uma interacção harmoniosa e económica do sistema musculo-esquelético, do sistema nervoso e sensorial com a finalidade de produzir acções motoras precisas, equilibradas e reacções rápidas adaptadas a diferentes situações s $^{15,16}$. Estabelecemos dois propósitos fundamentais: (1) chamar a atenção para a problemática dos efeitos a distância do BPN em crianças e jovens que são objecto de intervenção pedagógica - educação física e desporto - que busca eficiência em diferentes domínios da sua performance desportivo-motora; (2) apresentar aspectos relevantes do quadro conceptual da programação fetal e da sua relevância interpretativa na insuficiência do desempenho motor.

\section{PROCEDIMENTOS METODOLÓGICOS}

Para ter acesso à informação mais relevante, foi realizada uma pesquisa na base de dados Pubmed por meio de uma estratégia de busca com palavras-chave e suas possíveis combinações. Fontes adicionais foram obtidas através das referências encontradas nos artigos primários.

Para o primeiro propósito deste estudo, foram seleccionados 14 estudos (tranversais, longitudinais e caso-controlo) a partir de 1990 nos quais estão referenciados métodos e resultados da avaliação da performance motora, através das palavras-chave: BPN, desempenho motor e performance motora. Para o segundo objectivo, foram seleccionados 9 estudos fundamentais da programação fetal que podem perturbar o processo natural de desenvolvimento; foram seleccionados mediante as palavras-chave programação fetal, origens fetais e desenvolvimento fetal.

\section{PROBLEMÁTICA DOS EFEITOS DO BPN NA INTERVENÇÃO PEDAGÓGICA - EDUCAÇÃO FÍSICA E DESPORTO - QUE BUSQUE EFICIÊNCIA EM DIFERENTES DOMÍNIOS DA PERFORMANCE DESPORTIVO-MOTORA DE CRIANÇAS E JOVENS}

A entrada de cada criança nas primeiras etapas do lato universo do ciclo de ensino, desde o Jardim de infância até o início do $2^{\circ}$ ciclo implica, entre outros domínios de acção educativa, um conhecimento mais preciso do corpo, das suas funções e capacidades. Um papel importante e decisivo nestas etapas multifacetadas do desenvolvimento está atribuído à disciplina de Educação Física. A relevância e riqueza do seu conteúdo programático vão muito além do refinamento das habilidades motoras fundamentais. Ainda que a estruturação didáctico-metodológica das aulas esteja impregnada de um forte cariz lúdico assente no jogo, é importante perceber que é no ensino-aprendizagem de técnicas corporais de forte raiz cultural que se preenche, também, o espaço das aulas. Qualquer que seja o intervalo etário de intervenção pedagógica, as mais diversas tarefas motoras abordadas nas aulas reclamam um sentido próprio de aprimoramento, de sucesso e de realização. Estamos a falar da qualidade de desempenho motor. Não obstante alguma variação terminológica e delimitação conceptual para descrever o desempenho motor, o facto mais evidente é que as mais diversas tarefas motoras do quotidiano das crianças e jovens são executadas com níveis distintos de proficiência.

Esta variabilidade intraindividual e entre indivíduos é normalmente explicada em termos de factores genéticos e ambientais. Uma parte substancial de reduzida eficiência no desempenho é também condicionada, nalguns casos, por múltiplos aspectos decorrentes do BPN ${ }^{12,17,18}$. Se dirigirmos o nosso olhar exclusivamente para o grupo de crianças e jovens com BPN em diferentes países, é possível apresentar, genericamente, os seguintes dados essenciais cujos detalhes estão expressos na Tabela 1.

Dos 14 estudos que atenderam aos critérios de inclusão, 7 foram realizados em populações europeias $^{10,18-23}$, 6 na população norte america$\mathrm{na}^{7,9,11,12,24,25}$, e 1 na população australiana ${ }^{26}$. $\mathrm{Na}$ população europeia, os valores dos défices motores variaram entre $4 \%^{23}$ e $51 \%{ }^{21}$. A população norte americana apresentou valores que se situam entre $4 \%$ e $56 \%{ }^{24}$. Uma pesquisa realizada com a população da Austrália, com 255 elementos, encontrou um défice de coordenação motora para EBPN de 9,5\% ${ }^{26}$. Os estudos norte americanos salientaram, também, a componente da limitação funcional e de independência que variaram entre $15,5 \%^{7}$ e $81 \%{ }^{11}$, enquanto que os estudos europeus abordaram mais a limitação motora.

Algumas pesquisas recorreram, ainda, a delineamentos longitudinais ${ }^{9-11,20,21}$. Os estudos escolhidos usaram diversas baterias de testes, sendo as mais usuais (1) o Test of Motor Impairment (TOMI) 21,22 
e (2) o Movement Assessment Battery for Children (M-ABC) $)^{19,21,23,26}$. Alguns optaram por usar somente questionários, ${ }^{9,10,11,18}$ e outro o Peabody Developmental Motor Scale (PDMS) ${ }^{24}$.

Não obstante alguma diversidade metodológica, destaca-se nos resultados a presença de alterações significativas das capacidades motoras em crianças de BPN, com maior evidência nas idades $10 / 14^{9,10}$ e mantidas até idades mais tardias, mas que tendem a melhorar com o avançar da idade $^{19,20}$. Os estudos prévios monstraram que os problemas motores estão intimamente associados não só com dificuldades na performance escolar ${ }^{11,25}$, mas também com problemas emocionais e sociais ${ }^{18}$.

O DM é de vital relevância em diferentes domínios, tal como os estudos demonstraram, do biológico ao psicológico, influenciando as crianças e jovens de BPN na sua interacção com o meio ambiente, reflectindo-se quer em actividades de coordenação como também no ensino-aprendizagem. Da mesma forma, podem ter dificuldades em alcançar os seus objectivos ou satisfazer as suas necessidades pessoais, com consequências no relacionamento com os outros, com si próprio e do que é capaz de fazer.

Não há dúvida que,existem efeitos do BPN na intervenção pedagógica - educação física e desporto, com alteração da qualidade do desempenho motor, no entanto, sabe-se que, quanto maior for o peso ao nascer menor será a magnitude dos défices e também, que com o avançar da idade estes diminuem.

\section{ASPECTOS RELEVANTES DO QUADRO CONCEPTUAL DA PROGRAMAÇÃO FETAL E DA SUA RELEVÂNCIA INTERPRETATIVA NA INSUFICIÊNCIA DE DESEMPENHO MOTOR E COORDENATIVO}

Durante as últimas décadas, tem crescido o número de estudos que propõem diferentes mecanismos para explicar as bases biológicas das associações observadas entre o BPN e os resultados encontrados em estudos epidemiológicos, sobretudo, relacionados com factores de risco de doença cardiovascular.

$\mathrm{Nee}^{27}$ propôs a ideia da presença de "thrifty genes" que são seleccionados durante a evolução e cuja "responsabilidade" é actuar em mecanismos da saciedade/fome quando as reservas de alimento são escassas. Forsdahl ${ }^{28}$ introduziu a ideia de que a pobreza provoca má nutrição e condiciona aspectos da gestação das parturientes, induzindo vulnerabilidade a longo prazo nas crianças que sofreram deste constrangimento nutricional. Wadsworth et al. ${ }^{29}$ concluiram que a pressão sanguínea em adultos varia inversamente com o peso à nascença, o que parece suportar a proposta de Forsdahl ${ }^{28}$.

Lucas $^{30}$ avançou a hipótese da existência de adaptações embrionárias e fetais a um ambiente sub-optimal intrauterino que resulta em consequências permanentes. Esta "programação por indutores", i.e., estímulos iniciais ou "insultos" operam em períodos sensíveis do desenvolvimento pré-natal e se expressam em consequências embrionárias a longo prazo. Daqui a suposta existência de um sistema de "programação" que ocorre quando existe uma agressão ou estímulo precoce, que resultará em alterações permanentes a longo prazo ao nível estrutural ou funcional do organismo.

Em 1992, Hales et al. ${ }^{31}$ propõem a hipótese do "thrifty phenotype", uma extensão do "thrifty genotype", sugerindo que quando o ambiente fetal é pobre, existe sempre uma resposta adaptativa cujo resultado a distância temporal é a do BPN. Sendo a hipótese de programação a resposta mais plausível para a associação entre BPN e saúde, é denominada como o processo em que o estímulo ou agressão, em períodos críticos do desenvolvimento, teria repercussão na estrutura, na função de órgãos, tecidos e sistemas orgânicos. Barker ${ }^{13}$ cunhou a noção de "Origens Fetais" para diferenciar efeitos que podem ocorrer no período de crescimento fetal e que estão comprometidos em diferentes períodos de gestação.

Ben-Shlomo e $\mathrm{Kur}^{32}$ apresentaram uma proposta que seria complementar à hipótese de Barker e seus colaboradores, considerando que são importantes não apenas os estímulos ou agressões ocorridas na vida intrauterina, mas também a acumulação de riscos ao longo da vida. Esta concepção de risco seria diferente da "programação" por não requerer a existência de períodos críticos.

Waterland et al..$^{33}$ estenderam o conceito de "programação" a um domínio que denominam de "impressão metabólica" para descrever os fenómenos biológicos básicos que estão na base de relações entre experiências nutricionais precoces e doenças posteriores. Trata-se de identificar respostas adaptativas a condições específicas de natureza nutricional no início da vida e acompanhar o desenvolvimento de mecanismos subjacentes. A noção de impressão metabólica é caracterizada por diferentes aspectos: (1) ser uma janela crítica ontogénica de susceptibilidade limitada ao desenvolvimento inicial; (2) de efeito persistente até à fase adulta; (3) com resultados específicos e mensuráveis (que podem variar quantitativamente entre os indivíduos) e (4) uma resposta doseada ou 
Quadro 1. Método e resultados dos estudos realizados sobre insuficiência em diferentes domínios inclusive da performance desportivo-motora de crianças e jovens de BPN.

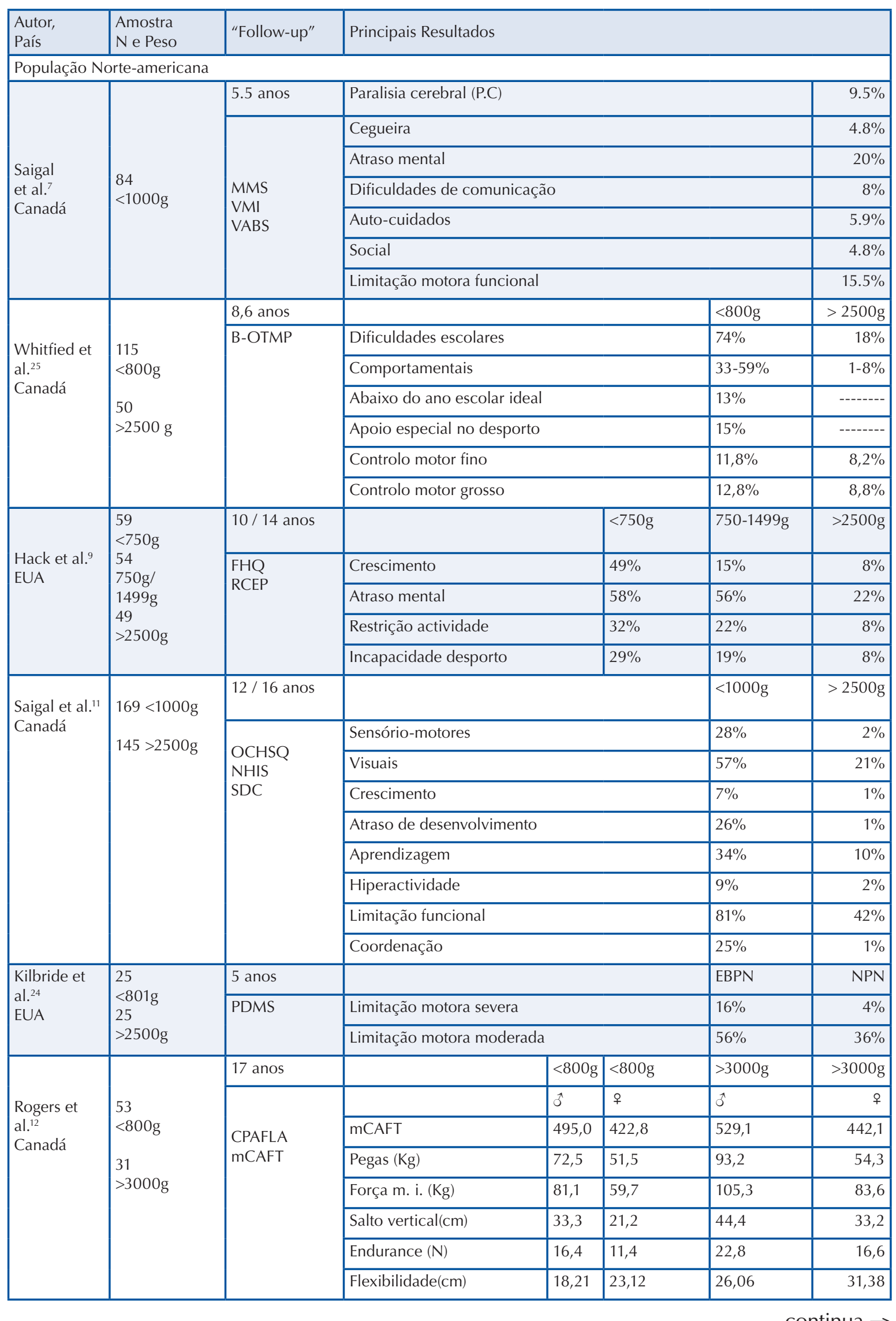


continua $\rightarrow$

\begin{tabular}{|c|c|c|c|c|c|c|}
\hline \multicolumn{7}{|c|}{ População Europeia } \\
\hline \multirow{4}{*}{$\begin{array}{l}\text { Pharoah et } \\
\text { al. }{ }^{24} \\
\text { Inglaterra }\end{array}$} & \multirow{4}{*}{$\begin{array}{l}233<2000 g \\
46>2500 g\end{array}$} & 8 anos & Casos/ controlo & $<1000 \mathrm{~g}$ & 1001-1500g & $\begin{array}{r}1501- \\
2000 \mathrm{~g}\end{array}$ \\
\hline & & \multirow{3}{*}{$\begin{array}{l}\text { TOMI } \\
\text { WISC }\end{array}$} & Sem défices & $44-81 \%$ & $67-87,5 \%$ & $\begin{array}{r}75,5- \\
94,5 \% \\
\end{array}$ \\
\hline & & & Défices moderados & $43-17 \%$ & $22-11,5 \%$ & $11,5-4 \%$ \\
\hline & & & Défices severos & $12,5-0 \%$ & $11,5-0,5 \%$ & $\begin{array}{l}13,5- \\
1,5 \%\end{array}$ \\
\hline \multirow{3}{*}{$\begin{array}{l}\text { Powls et al. }{ }^{21} \\
\text { Inglaterra }\end{array}$} & \multirow{3}{*}{$\begin{array}{l}47<1250 \mathrm{~g} \\
60>2500 \mathrm{~g}\end{array}$} & $8,12 / 13$ anos & & & $<1250 \mathrm{~g}$ & $>2500 \mathrm{~g}$ \\
\hline & & \multirow{2}{*}{$\begin{array}{l}\text { TOMI } \\
\text { M-ABC }\end{array}$} & \multicolumn{2}{|l|}{8 anos } & $34 \%$ & $5 \%$ \\
\hline & & & \multicolumn{2}{|l|}{ 12/13 anos } & $51 \%$ & $8,5 \%$ \\
\hline \multirow{4}{*}{$\begin{array}{l}\text { Jongsmans } \\
\text { et al. }{ }^{23} \\
\text { Inglaterra }\end{array}$} & \multirow{4}{*}{$\begin{array}{l}183<2500 \mathrm{~g} \\
215 \\
>2500 \mathrm{~g}\end{array}$} & 6 anos & & $<1000 \mathrm{~g}$ & $\begin{array}{l}1000 / \\
1499 g\end{array}$ & $\begin{array}{r}1500 / \\
2499 g\end{array}$ \\
\hline & & \multirow{3}{*}{$\begin{array}{l}\text { TE } \\
\text { M-ABC } \\
\text { VMI }\end{array}$} & $\mathrm{TE}$ & $35 \%$ & $38 \%$ & $38 \%$ \\
\hline & & & $M-A B C$ & $9 \%$ & $5,3 \%$ & $4 \%$ \\
\hline & & & VMI & $7 \%$ & $8 \%$ & $9 \%$ \\
\hline \multirow{5}{*}{$\begin{array}{l}\text { Walther et } \\
\text { al. }^{10} \\
\text { Holanda }\end{array}$} & \multirow{5}{*}{$1097<1500 \mathrm{~g}$} & $\begin{array}{l}2,5 \text { e } 9 / 14 \\
\text { anos }\end{array}$ & anos & $\begin{array}{l}\text { Défices } \\
\text { severos }\end{array}$ & \begin{tabular}{|l} 
Défices \\
ligeiros
\end{tabular} & Inaptidão \\
\hline & & \multirow{4}{*}{$\begin{array}{l}\text { Questionários } \\
\text { informais }\end{array}$} & 2 & $6,3 \%$ & $12,0 \%$ & -------- \\
\hline & & & 5 & $6,9 \%$ & $8,4 \%$ & $13,2 \%$ \\
\hline & & & 9 & $10,1 \%$ & $16,6 \%$ & $18,3 \%$ \\
\hline & & & 14 & $6,3 \%$ & $15,9 \%$ & $29,0 \%$ \\
\hline \multirow{9}{*}{$\begin{array}{l}\text { Cooke }^{18} \\
\text { Inglaterra }\end{array}$} & \multirow{9}{*}{$\begin{array}{l}138<1500 g \\
163>2500 g\end{array}$} & $6 / 12$ anos & & & $<1500 \mathrm{~g}$ & $>2500 \mathrm{~g}$ \\
\hline & & \multirow{8}{*}{$\begin{array}{l}\text { SF-36 } \\
\text { HADS }\end{array}$} & \multicolumn{2}{|l|}{ Função física } & $14,3 \%$ & $10,0 \%$ \\
\hline & & & \multicolumn{2}{|l|}{ Limitação física } & $30,2 \%$ & $26,2 \%$ \\
\hline & & & \multicolumn{2}{|l|}{ Emocional } & $32,3 \%$ & $39,0 \%$ \\
\hline & & & \multicolumn{2}{|l|}{ Social } & $23,7 \%$ & $21,8 \%$ \\
\hline & & & \multicolumn{2}{|l|}{ Saúde mental } & $19,9 \%$ & $21,1 \%$ \\
\hline & & & \multicolumn{2}{|l|}{ Energia } & $22,1 \%$ & $22,5 \%$ \\
\hline & & & \multicolumn{2}{|l|}{ Dor } & $23,2 \%$ & $17,6 \%$ \\
\hline & & & \multicolumn{2}{|l|}{ Percepção de saúde } & $23,3 \%$ & $20,5 \%$ \\
\hline \multirow[b]{2}{*}{$\begin{array}{l}\text { Eversen et } \\
\text { al. }{ }^{19} \\
\text { Noruega }\end{array}$} & \multirow{2}{*}{$\begin{array}{l}54<1500 g \\
59 \\
\text { SGA } \\
83>2500 g\end{array}$} & 14 anos & & $<1500 \mathrm{~g}$ & SGA & $>2500 \mathrm{~g}$ \\
\hline & & $M-A B C$ & Défices motores & $11 \%$ & $7,3 \%$ & $6,3 \%$ \\
\hline & & 8 e 15 anos & & & 8 anos & 15 anos \\
\hline $\begin{array}{l}\text { O'Brien et } \\
\text { al. }{ }^{20}\end{array}$ & $\begin{array}{l}207 \text { e } \\
152\end{array}$ & WISC-R & Normal & & $82 \%$ & $66 \%$ \\
\hline & $<2230 g$ & $\begin{array}{l}V M I \\
\text { K-ABC }\end{array}$ & Défices motores & & $13 \%$ & $30 \%$ \\
\hline & & & Inaptidão & & $5 \%$ & $4 \%$ \\
\hline População Au & istraliana & & & & & \\
\hline Davis et al. ${ }^{26}$ & 255 & 8,8 anos & & & $<1000 \mathrm{~g}$ & $>2500 \mathrm{~g}$ \\
\hline Austrália & $<1000 \mathrm{~g}$ & M-ABC & Défices de coordenação & & $9,5 \%$ & $2 \%$ \\
\hline
\end{tabular}

TE: Touwen's Examination of the Child with Minor Neurological Dysfunction; M-ABC: Movement Assessment Battery for Children; VMI: Developmental Test of Visual-Motor Integration; CPAFLA: Canadian Physical Activity, Fitness and Lifestyle Appraisal; mCAFT: Modified Fitness Test; MMS: McCarthy Motor Scale; VABS: Vineland Adaptative Behavior Scales; FHQ: Family and Health Questionnaire; RCEP: Report of the Child's Educational Progress; BOTMP: Bruininks-Oseretsky Test of Motor Proficiency; TOMI: Test of Motor Impairment; OCHSQ: Ontario Child Health Study Questionnaire; NHIS: National Health Interview Survey; SDC: Survey of Disabled Children; WISC-R: Wechsler Intelligence Scales for Children-revised; K-ABC: Kaufman Assessment Battery for Children; HADS: Hospital Anxiety and Depression Scale; SF-36: Short Form 36 Health Survey; SGA: Small for Gestational Age; PDMS: Peabody Developmental Motor Scale; NPN: Normal Peso ao Nascer; MI: membro inferior. 
relação entre um limite específico de exposição e seu resultado.

Nasce daqui a possibilidade de identificar cinco mecanismos decorrentes: (1) induzir variações na estrutura de um órgão, (2) na alteração no número de células, (3) na selecção clonal, (4) na remodelação apoptótica e (5) na diferenciação metabólica. Todos estes mecanismos podem ocorrer antes e durante o período fetal.

Em 2004, Barker ${ }^{34}$ propôs a expressão "Developmental Plasticity" ao invés de "programação" para expressar a capacidade de um único genótipo em produzir mais de uma forma alternativa de estruturas, estados fisiológicos ou comportamentais em resposta a condições ambientais. Também foi proposto por Gluckman et al. ${ }^{35}$, a noção de respostas homeostáticas que representam adaptações fetais com alterações no ambiente intrauterino e que podem ter consequências a longo termo - a natureza da resposta é determinada pelo ambiente pós-natal previsto e real. O feto apresenta uma gama de definições homeostáticas para a vida pós-natal, adequado de acordo com as informações que recebe no útero. Se o ambiente pós-natal corresponde ao real da previsão pré-natal, o risco da doença é baixo, se eles não corresponderem, o risco da doença é maior, e daqui a expressão "Predictive Adaptive".

A maior fatia da investigação empírica sobre programação fetal é de natureza descritiva, em humanos, e cuja expressão decorre da forma de pensar da Epidemiologia, não obstante alguma investigação no modelo animal ${ }^{36}$. A pesquisa descrita pelos autores mencionados neste ponto versa, sobretudo, a associação entre BPN com doenças cardiovasculares, incluindo hipertensão arterial, tolerância à glicose, diabetes tipo-2, resistência à insulina e obesidade. Também foram objecto de estudo o acidente vascular cerebral (AVC), a doença obstrutiva do pulmão, o tumor nos ovários, o suicídio, o tiroidismo autoimune e a falência renal.

Apesar da popularidade e plausibilidade das teorias e informação disponível, Kramer ${ }^{37}$ sugere a possibilidade da eventual existência de problemas na interpretação causal, uma vez que nem sempre se controla os efeitos de selecção das amostras relacionados aos aspectos de natureza sócioeconómica dos sujeitos e dos métodos de análise dos resultados obtidos.

Mcmillen et al. ${ }^{36}$ afirmam que para além das doenças anteriormente descritas, a hipótese de "origens fetais" evidenciou, mediante estudos experimentais, que alterações ao nível molecular, celular, metabólico, neuro-endócrino e adaptações fisiológicas resultam em alterações permanentes no padrão do desenvolvimento da proliferação e diferenciação celular em tecidos ou órgãos que induzem consequências patológicas tardias. Há quem refira uma influência directa no desenvolvimento músculo-esquelético ${ }^{38}$.

Não obstante a relevância da marcha sequencial das ideias do "thrifty phenotype" até à "Developmental Plasticity", o facto é que a atenção primordial das explicações putativas se referem a doenças metabólicas. Não são conhecidos aspectos dos eventuais mecanismos que em condições adversas no desenvolvimento fetal induzirão problemas no desenvolvimento neuromotor de crianças, jovens e adultos, e que não possam ser "compensados" com estimulação sensório-motora em diferentes momentos da vida pós-natal.

\section{CONSIDERAÇÕES FINAIS}

Os avanços médicos e técnicos nas unidades neonatais contribuíram para um aumento da sobrevida de crianças com BPN e prematuras, mas também aumentou a sua morbilidade.

As crianças e jovens com BPN apresentam, inquestionavelmente, diversas alterações, algumas bem adversas, no seu DM, cognitivo, social, escolar, entre outros. Contudo, não existe conclusão definitiva acerca da sua extensão, reversibilidade e significado face à enorme variabilidade dos valores dos défices. A previsão, ao nível individual, dos problemas que enfrentarão no seu desenvolvimento futuro continua a ser uma tarefa bem difícil.

Os investigadores são confrontados com o desafio de elucidar os mecanismos específicos que estão subjacentes à persistência ou não de alterações relevantes do quadro conceptual da programação fetal e da sua relevância interpretativa na insuficiência de desempenho motor e coordenativo, sendo esta uma tarefa complexa. Na verdade, a persistência de efeitos, em sistemas distintos, torna difícil a determinação do(s) mecanismo(s) mais dominantes. Um tema recorrente na fisiologia do desenvolvimento é que, embora o impacto inicial de um evento desencadeante possa ser relativamente subtil, os seus efeitos poderão ser ampliados ao longo do tempo, mas isso não significa que o evento pré-natal seja o único responsável. A importância de ganhar esta informação é grande, especialmente, entre populações que continuam a experienciar altas taxas de crianças com BPN.

Parece ser consensual que as influências intrauterinas durante períodos críticos do desenvolvimento humano podem ter implicações a 
longo-prazo. Há quem avance a hipótese da indução de alterações no ciclo de vida de um indivíduo provocando lesões, algumas irreversíveis. Não obstante a relevância da marcha sequencial das ideias do "thrifty phenotype" até à "Developmental Plasticity", o facto é que a atenção primordial das explicações se referem às doenças metabólicas. Não são conhecidos aspectos dos eventuais mecanismos que, em condições adversas no desenvolvimento fetal, induzirão problemas no desenvolvimento neuromotor de crianças, jovens e adultos, e que não possam ser "compensados" com estimulação sensório-motora em diferentes momentos da vida pós-natal. A avaliação dos resultados dos factores que estão na origem do BPN continua a ser um desafio estimulante para investigadores de todo mundo, principalmente, dos países em desenvolvimento.

\section{REFERÊNCIAS BIBLIOGRÁFICAS}

1. Malina RM, Bouchard C. Growth, Maturation and Physical Activity. Champaign: Human Kinetics, 2004.

2. Wardlaw T, Blanc A, Zupan J, Åhman E. Low Birthweight Country, Regional and Global Estimates [online]. New York, United States: UNICEF and WHO Digital Library; 2004. Available from: <http:// www .who.int/ reproductive health/publications low_birthweight/ low_birthweight_estimates.Pdf $>$.[2008 Jan 20].

3. Blackman JA. Neonatal Intensive Care: is it worth it? Developmental Sequelae of very Low Birthweight. Pediatr Clin N Am 1991;38(6):1497-511.

4. Msall ME, Tremont MR. Measuring functional outcomes after prematurity: developmental impact of very low birth weight and extremely low birth weight status on childhood disability. Ment Retard Dev Disabil Res Rev 2002;8:258-72.

5. Wolf MJ, Koldewijn K, Beelen A, Smit B, Hedlund R, de Groot IJM. Neurobehavioral and developmental profile of very low birthweight preterm infants in early infancy. Acta Paediatr 2002;91(8):930-8.

6. Russell RB, Green NS, Steiner CA, Meikle S, Howse JL, Poschman K, et al. Cost of Hospitalization for Preterm and Low Birth Weight Infants in the United States. Pediatrics 2007;120(1):1-9.

7. Saigal S, Szatmari P, Rosenbaum P, Campbell D, King S. Intellectual and functional status at school entry of children who weighed 1000 grams or less at birth: A regional perspective of births in the 1980s. J Pediatr 1990;116(3):409-16.

8. Palta M, Sadek-Badawi M, Evans M, Weinstein MR, McGuinness G. Funcional assessment of a multicenter very low birthweight cohort at age 5 years. Arch Pediatr Adolesc Med 2000;154(1):23-30.

9. Hack M, Taylor HG, Klein N, Mercuri-Minich N. Functional limitations and special health care needs of 10 to 14 years old children weighing less than $750 \mathrm{~g}$ at birth. Pediatrics 2000;106(3):554-60.
10. Walther FJ, den Ouden AL, Verloove-Vanhorick SP. Looking back in time: outcome of a national cohort of very preterm infants born in The Netherlands in 1983. Early Hum Dev 2000;59(3):175-91.

11. Saigal S, Stoskopf BL, Streiner DL, Burrows E. Physical Growth and Current Health Status of Infants Who Were of Extremely Low Birth Weight and Controls at Adolescence. Pediatrics 2001;108(2):407-15.

12. Rogers M, Fay TB, Whitfield MF, Tomlinson J, Grunan RE. Aerobic Capacity, strength, flexibility, and activity level in unimpaired extremely low birthweight $(<800 \mathrm{~g})$ survivors at 17 years of age. Compared with term-born control subjects. Pediatrics 2005;116(1):58-65.

13. Barker DJP. Fetal origins of coronary heart disease. BMJ 1995;311(6998):171-4.

14. Loos R. Fetal origins of cardiovascular and metabolic risk factors in young adults. A prospective twin stud. $\mathrm{PhD}$ [Dissertation in Faculty of Medicine Center for Human Genetics]. Leuven: Katholieke Universiteit; 2000.

15. Kiphard EJ, Schilling JJ. KorperKoordination-test fur kinder, KTK. Weiheim, Beltz 1974.

16. Kiphard EJ. Insuficiencias de movimiento y de coordinación en la edad de la escuela primaria. Buenos Aires: Kapelusz; 1976.

17. Rosenbaum P, Saigal S, Szatmari P, Hoult L. Vineland Adaptive Behavior Scales as a summary of functional outcome of extremely low-birthweight children. Dev Med Child Neurol 1995;37(7):577-86.

18. Cooke RWI. Health, lifestyle, and quality of life for young adults born very preterm. Arch Dis Child 2004;89:201-6.

19. Evensen KAI, Vik T, Helbostad J, Indeadavik MS, Kulseng S, Brubakk AM. Motor Skills in adolescents with low birthweight. Arch Dis Child 2004;89:451-5.

20. O’Brien F, Roth S, Stewrt A, Rifkin L, Rushe T, Wayatt J. The neurodevelopmental progress of infants less than 33 weeks into adolescence. Arch Dis Child, 2004;89(3):207-211.

21. Powls A, Botting N, Cooke RWI, Marlow N. Motor impairment in children 12 to 13 years old with a birthweight of less than 1250g. Arch Dis Child, 1995;73(2):62-6.

22. Pharoah PO, Stevenson CJ, Cooke RW, Stevenson $\mathrm{RC}$. Clinical and subclinical deficits at 8 years in a geographically defined cohort of low birthweight infants. Arch Dis Child 1994;70(4):264-70.

23. Jongmans M, Mercuri E, de Vries L, Dubowitz L, Henderson S. Minor neurological signs and perceptual-motor difficulties in prematurely born children. Arch Dis Child 1997;76(1):9-14.

24. Kilbride HW, Thorstad K, Daily DK. Preschool outcome of less than $801 \mathrm{~g}$ preterm infants compared with full-term siblings. Pedriatrics 2004;113(4):742-7.

25. Whitfield MF, Grunau RVE, Holsti L. Extremely premature $(<800 \mathrm{~g})$ schoolchildren: Multiple areas of hidden disability. Arch Dis Child Fetal Neonatal 1997;77(2):85-90.

26. Davis NM, Ford GW, Anderson PJ, Doyle LW. Developmental coordination disorder at 8 years of age in a regional cohort of extremely-low-birthweight of very preterm infants. Dev Med Child Neurol 2007;49(5):325-30. 
27. Neel JV. Diabetes mellitus: a "thrifty" genotype rendered detrimental by "progress"? Am J Hum Genet 1962;14:353-62.

28. Forsdahl A. Are poor living conditions in childhood and adolescence an important risk factor for arteriosclerotic heart disease? Br J Prev Soc Med 1977; 31(2):91-5.

29. Wadsworth ME, Cripps HA, Midwinter RE, Colley JR. Blood pressure in a national birth cohort at age of 36 related to social and familial factors, smoking and body mass. Br Med J 1985;291(6508):1534-8.

30. Lucas A. Programming by early nutrition in man. In the Childhood environment and adult disease. CIBA Foundation Symposium 156. Willey: Chicherster 1991, p.38-55.

31. Hales CN, Barker DJ. Type 2 (non-insulin-dependent) diabetes mellitus: the thrifty phenotype hypothesis. Diabetologia 1992,35(7):593-601.

32. Ben-Shlomo Y, Kuh D. A life course approach to chronic disease epidemiology: conceptual models, empirical challenges and interdisciplinary perspectives. Int J Epidemiol 2002;31:285-93.

33. Waterland RA, Garza C. Potencial mechanisms of metabolic imprinting that lead to chronic disease. Am J Clin Nutr, 1999;69(2):179-97.
34. Barker DJP. Developmental origins of adult health and disease. J Epidemiol Community Health 2004;58:114-5.

35. Gluckman PD, Hanson MA. The developmental origins of the metabolic syndrome. Trends Endocrinol Metab 2004;15(4):183-187.

36. McMillen IC, Robinson JS. Developmental origins of metabolic syndrome: prediction, plasticity and programming. Physiol Rev 2005;85:571-633.

37. Kramer M. Invited Commentary: Association between restricted fetal growth and adult chronic disease: is it causal? Is it important? Am J Epidemiol 2000;152(7):605-8.

38. Dwyer CM, Stickland NC. Does the anatomical location of a muscle affect the influence of undernutrition on muscle fiber number? J Anat 1992;181(2):373-6.

\section{Endereço para correspondência}

José António Ribeiro Maia

Rua Dr. Plácido Costa, 91

4200.450 Porto, Portugal

E-mail: jmaia@fade.up.pt 